\title{
Temperature dependence of critical micelle concentration of polyoxyethylenated non-ionic surfactants
}

\author{
Li-Jen Chen ${ }^{a, *}$, Shi-Yow Lin ${ }^{b, 1}$, Chiung-Chang Huang ${ }^{b}$, En-Ming Chen ${ }^{b}$ \\ a Department of Chemical Engineering, National Taiwan University, Taipei, Taiwan 106, People's Republic of China \\ b Department of Chemical Engineering, National Taiwan University of Science and Technology, Taipei, Taiwan 106, \\ People's Republic of China
}

Received 13 May 1997; accepted 13 August 1997

\begin{abstract}
The critical micelle concentrations (CMCs) of an $n$-dodecyl polyoxyethylene glycol monoether $\left(\mathrm{C}_{12} \mathrm{H}_{25} \mathrm{O}\left(\mathrm{C}_{2} \mathrm{H}_{4} \mathrm{O}\right)_{j} \mathrm{H}\right)$ with three different oxyethlyene chain length ${ }_{j}=4,6$ and 8$)$ are experimentally determined over the temperature ranging from $10^{\circ} \mathrm{C}$ to $80^{\circ} \mathrm{C}$ using the Wihelmy plate technique. It is found that there exists a minimum CMC in the CMC-temperature curve. The temperature of the minimum CMC for three systems is around $50^{\circ} \mathrm{C}$. The enthalpy and entropy of micelle formation are evaluated. The correlation of enthalpy and entropy of micelle formation exhibits an excellent linearity, and the compensation temperature is $321 \mathrm{~K}$, dramatically larger than previous findings for non-ionic surfactants in aqueous solutions. (c) 1998 Elsevier Science B.V.
\end{abstract}

Keywords: Surfactant; Surface tension; Critical micelle concentration; Wihelmy plate; Enthalpy-entropy compensation

\section{Introduction}

It is well known that the critical micelle concentration (CMC) varies with temperature. For ionic surfactants in aqueous solution, the CMC first monotonically decreases to a certain minimum with increasing temperature and then increases with further increase in temperature, that is there exists a minimum in the curve of CMC vs. temperature. For ionic surfactants in aqueous solution, the minimum in the CMC-temperature curve appears around $25^{\circ} \mathrm{C}$, according to the available data in literature [1-4]. However, for the nonionic surfactants, only $p, t$-octylphenol polyoxyethylene glycol monoether $\left(\mathrm{OPE}_{j}\right)$ in aqueous solu-

\footnotetext{
*Corresponding author. E-mail: ljchen@ccms.ntu.edu.tw

${ }^{1}$ Also corresponding author. E-mail: ling@ch.ntust.edu.tw
}

tion has been reported $[1,5]$, to the best of our knowledge, to also exhibit a minimum in the CMC-temperature curve. The temperature of the minimum $\mathrm{CMC}$ of $\mathrm{OPE}_{j} \mathrm{~s}$ in the $\mathrm{CMC}$-temperature curve increases as the oxyethylene chain length increases. The temperature of minimum $\mathrm{CMC}$ is around $50^{\circ} \mathrm{C}$ for the $\mathrm{OPE}_{j} \mathrm{~s}$ with oxyethylene chain length $j$ varying from 6 to 10 .

Although polyoxyethylenated non-ionic surfactants are extensively used, both in industrial applications and in fundamental research, the available data [6-9] indicate that there is a general trend for the polyoxyethylenated non-ionic surfactants in aqueous solution according to which the CMC decreases with temperature. However, most of these experiments were carried out only up to $45^{\circ} \mathrm{C}$. It is fair to assume that $45^{\circ} \mathrm{C}$ is too low to observe the minimum CMC behavior in the CMC-temperature curve. 
In this study, the n-dodecyl polyoxyethylene glycol monoether $\left(\mathrm{C}_{12} \mathrm{E}_{j}\right)$ with three different oxyethylene chain lengths $\left({ }_{j}=4,6\right.$ and 8$)$ are chosen to examine the temperature effect on the $\mathrm{CMC}$ over a wide temperature ranging from $10^{\circ} \mathrm{C}$ to $80^{\circ} \mathrm{C}$. $\mathrm{C}_{i} \mathrm{E}_{j}$ is the abbreviation of non-ionic surfactant $\mathrm{CH}_{3}\left(\mathrm{CH}_{2}\right)_{i-1} \mathrm{O}\left(\mathrm{CH}_{2} \mathrm{CH}_{2} \mathrm{O}\right)_{j} \mathrm{H}$. It is found that the minimum in the $\mathrm{CMC}$ vs. temperature curve also exists in the surfactant $\mathrm{C}_{12} \mathrm{E}_{j}$ in aqueous solutions. The enthalpy and entropy of micelle formation are then evaluated according to the phase separation model. We further discuss the enthalpy-entropy compensation phenomenon in the micellization process.

\section{Experimental}

Non-ionic surfactants $\mathrm{C}_{12} \mathrm{E}_{4}, \mathrm{C}_{12} \mathrm{E}_{6}$, and $\mathrm{C}_{12} \mathrm{E}_{8}$ were purchased from Nikko Chemicals Co., and used without further purification. Water was purified in a Barstead NANOpure II system with the resistance around $18 \mathrm{M} \Omega \mathrm{cm}$.

The surface tension measurements were made with a surface tensiometer (CBVP-A3, Face) and a dynamic contact angle analyzer (DCA322, Cahn), using a sand-blasted platinum plate of dimensions $1.95 \times 1.00 \times 0.02 \mathrm{~cm}^{3}$.

Solutions were kept in a double-walled Pyrex vessel thermostatically controlled at a prescribed temperature. To prevent the contamination of the solution from dust in the air during the operation, the vessel had a cover with a hole only allowing a thin wire (attached to the platinum plate) to go through, and the whole vessel was placed inside a closed sample chamber of the surface tensiometer or the dynamic contact angle analyzer.

Since it was found that the surface tension decreases with time, the measurements were carried out until an equilibrium was established. For certain systems, for example $2 \times 10^{-10} \mathrm{~mol} \mathrm{~cm}^{-3}$ $\mathrm{C}_{12} \mathrm{E}_{8}$ at $25^{\circ} \mathrm{C}$, this phenomenon was very pronounced, and it took more than $10 \mathrm{~h}$ to reach the equilibrium. It is believed that this dynamic behavior of surface tension is mainly due to the slow mass transfer of surfactant molecules from the bulk solution onto the interface and the slow rearrangement of surfactant molecular configura- tion at the interface. However, the recent experimental data of adsorption kinetics of $\mathrm{C}_{12} \mathrm{E}_{8}$ show that the impurity effect is insignificant in the surface tension relaxation process $[10,11]$.

\section{Results and discussion}

The surface tensions were measured as a function of surfactant concentration at several different temperatures, as shown in Fig. 1. The CMC was then taken as the concentration at the sharp break, as listed in Tables 1-3 for three surfactants. Note that at a constant temperature the CMC increases as the number of oxyethylene groups increases. Such behavior is directly related to the increase of hydrophilicity of the $\mathrm{C}_{i} \mathrm{E}_{j}$ molecules with increasing oxyethylene chain length.

For each surfactant, as the system temperature increases, the CMC initially decreases and then increases, as shown in Fig. 2. The initial decrease of the $\mathrm{CMC}$ with temperature is a consequence of the decreased hydrophilicity of the surfactant molecules, owing to the smaller probability of hydrogen bond formation at higher temperatures. In other words, the increase in temperature causes the decrease in hydration of the hydrophilic oxyethylene group, which favors micellization. Consequently, the onset of micellization occurs at lower concentrations as the temperature increases. On the other hand, while the surfactant molecules dissolve in water, the hydrophobic group distorts the water structure. The increase in temperature also causes the increase in breakdown of the structured water surrounding the hydrophobic alkyl group, which disfavors micellization. Consequently, the onset of micellization tends to occur at higher concentrations as the temperature increases. Therefore, as the temperature increases further, the effect of the hydrophobic groups begins to exert an influence and finally predominates as the $\mathrm{CMC}$ reaches a minimum value and finally increases with temperature.

The temperatures of minimum CMC are found to be $320 \mathrm{~K}, 323 \mathrm{~K}$ and $325 \mathrm{~K}$ for the surfactants $\mathrm{C}_{12} \mathrm{E}_{4}, \mathrm{C}_{12} \mathrm{E}_{6}$, and $\mathrm{C}_{12} \mathrm{E}_{8}$ respectively. Note that the temperature of minimum $\mathrm{CMC}$ is systematically higher as the oxyethylene chain length is 

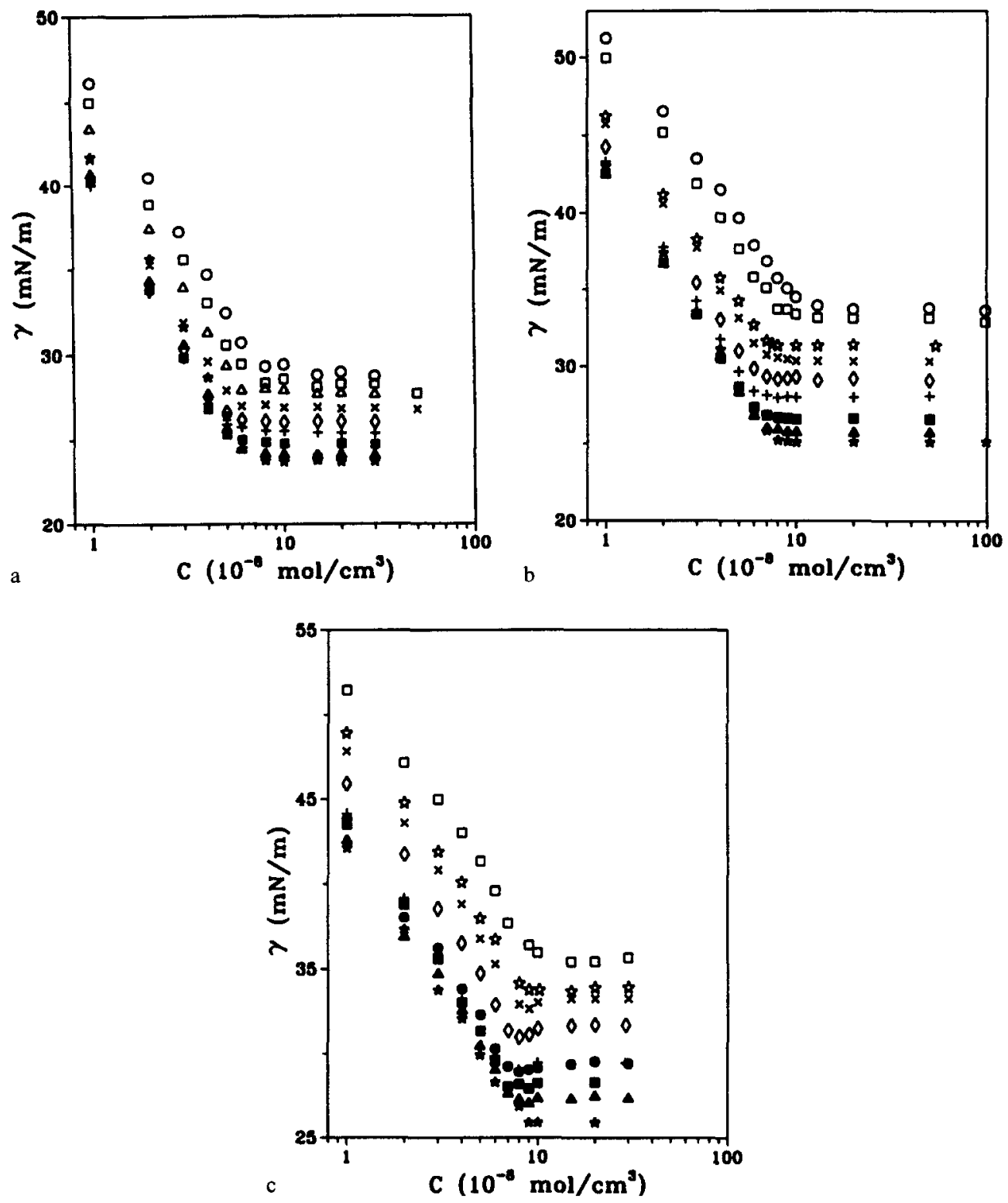

Fig. 1. The variation of surface tension as a function of surfactant concentration in aqueous solution (a) $C_{12} E_{4},(b) C_{12} E_{6}$, and (c) $\mathrm{C}_{12} \mathrm{E}_{8}$ at different temperatures: $10^{\circ} \mathrm{C}(\bigcirc), 15^{\circ} \mathrm{C}(\square), 20^{\circ} \mathrm{C}(\triangle), 25^{\circ} \mathrm{C}($ 市 $), 30^{\circ} \mathrm{C}(\times), 40^{\circ} \mathrm{C}(\diamond), 50^{\circ} \mathrm{C}(+), 55^{\circ} \mathrm{C}(\bullet), 60^{\circ} \mathrm{C}(\boldsymbol{\square})$, $70^{\circ} \mathrm{C}(\Delta)$, and $80^{\circ} \mathrm{C}(\star)$.

longer, in accord with the result of Crook et al. [5] for $\mathrm{OPE}_{j} \mathrm{~s}$ in aqueous solution.

Surface tensions of the surfactant solution $\gamma_{\mathrm{CMC}}$ at and above the CMC at different temperatures are also listed in Tables 1-3. It is obvious that for all three surfactant solutions the surface tension $\gamma_{\text {CMC }}$ decreases monotonically as the temperature increases, and $\mathrm{C}_{12} \mathrm{E}_{4}$ is the most surface active among the three systems over the whole temperature range. It should be further noted that the decrease in surface tension $\gamma_{\text {CMC }}$ is more pronounced for surfactants with a longer oxyethylene 
Table 1

Experimental results of $\mathrm{CMC}$ and surface tension at $\mathrm{CMC}$ for surfactant $\mathrm{C}_{12} \mathrm{E}_{4}$

\begin{tabular}{llll}
\hline$T\left({ }^{\circ} \mathrm{C}\right)$ & $\begin{array}{l}\mathrm{CMC} \\
\left(\mathrm{mol} \mathrm{cm}^{-3}\right)\end{array}$ & $\begin{array}{l}\gamma_{\mathrm{CMC}} \\
\left(\mathrm{mN} \mathrm{m}^{-1}\right)\end{array}$ & $\begin{array}{l}\pi_{\mathrm{CMC}} \\
\left(\mathrm{mN} \mathrm{m}^{-1}\right)\end{array}$ \\
\hline 10 & $7.2 \times 10^{-8}$ & 28.9 & 45.4 \\
15 & $6.5 \times 10^{-8}$ & 28.1 & 45.4 \\
20 & $6.0 \times 10^{-8}$ & 27.8 & 45.0 \\
30 & $5.3 \times 10^{-8}$ & 26.9 & 44.3 \\
40 & $4.7 \times 10^{-8}$ & 26.1 & 43.5 \\
50 & $4.8 \times 10^{-8}$ & 25.4 & 42.5 \\
60 & $4.9 \times 10^{-8}$ & 24.8 & 41.4 \\
70 & $5.8 \times 10^{-8}$ & 24.2 & 40.2 \\
80 & $6.4 \times 10^{-8}$ & 23.7 & 38.9 \\
\hline
\end{tabular}

Table 2

Experimental results of $\mathrm{CMC}$ and surface tension at $\mathrm{CMC}$ for surfactant $\mathrm{C}_{12} \mathrm{E}_{6}$

\begin{tabular}{llll}
\hline$T\left({ }^{\circ} \mathrm{C}\right)$ & $\begin{array}{l}\mathrm{CMC} \\
\left(\mathrm{mol} \mathrm{cm}{ }^{-3}\right)\end{array}$ & $\begin{array}{l}\gamma_{\mathrm{CMC}} \\
\left(\mathrm{mN} \mathrm{m}^{-1}\right)\end{array}$ & $\begin{array}{l}\pi_{\mathrm{CMC}} \\
\left(\mathrm{mN} \mathrm{m}^{-1}\right)\end{array}$ \\
\hline 10 & $1.0 \times 10^{-7}$ & 33.7 & 40.5 \\
15 & $8.6 \times 10^{-8}$ & 33.1 & 40.4 \\
25 & $7.2 \times 10^{-8}$ & 31.4 & 40.6 \\
30 & $6.8 \times 10^{-8}$ & 30.4 & 40.8 \\
40 & $6.2 \times 10^{-8}$ & 29.2 & 40.4 \\
50 & $6.0 \times 10^{-8}$ & 28.0 & 39.9 \\
60 & $6.2 \times 10^{-8}$ & 26.6 & 39.6 \\
70 & $6.5 \times 10^{-8}$ & 25.8 & 38.6 \\
80 & $7.3 \times 10^{-8}$ & 25.1 & 37.5 \\
\hline
\end{tabular}

Table 3

Experimental results of $\mathrm{CMC}$ and surface tension at $\mathrm{CMC}$ for surfactant $\mathrm{C}_{12} \mathrm{E}_{8}$

\begin{tabular}{llll}
\hline$T\left({ }^{\circ} \mathrm{C}\right)$ & $\begin{array}{l}\mathrm{CMC} \\
\left(\mathrm{mol} \mathrm{cm}^{-3}\right)\end{array}$ & $\begin{array}{l}\gamma_{\mathrm{CMC}} \\
\left(\mathrm{mN} \mathrm{m}^{-1}\right)\end{array}$ & $\begin{array}{l}\pi_{\mathrm{CMC}} \\
\left(\mathrm{mN} \mathrm{m}^{-1}\right)\end{array}$ \\
\hline 15 & $9.7 \times 10^{-8}$ & 35.5 & 38.0 \\
25 & $8.4 \times 10^{-8}$ & 33.9 & 38.1 \\
30 & $7.6 \times 10^{-8}$ & 33.3 & 37.9 \\
40 & $6.8 \times 10^{-8}$ & 31.7 & 37.9 \\
50 & $6.5 \times 10^{-8}$ & 29.5 & 38.4 \\
55 & $6.8 \times 10^{-8}$ & 29.4 & 37.6 \\
60 & $6.9 \times 10^{-8}$ & 28.2 & 38.0 \\
70 & $7.2 \times 10^{-8}$ & 27.4 & 37.0 \\
80 & $9.0 \times 10^{-8}$ & 25.9 & 36.7 \\
\hline
\end{tabular}

chain length, as shown in Fig. 3, due to an increase of the rupture of hydrogen bonds. It is well understood that there exists hydrogen bonding between

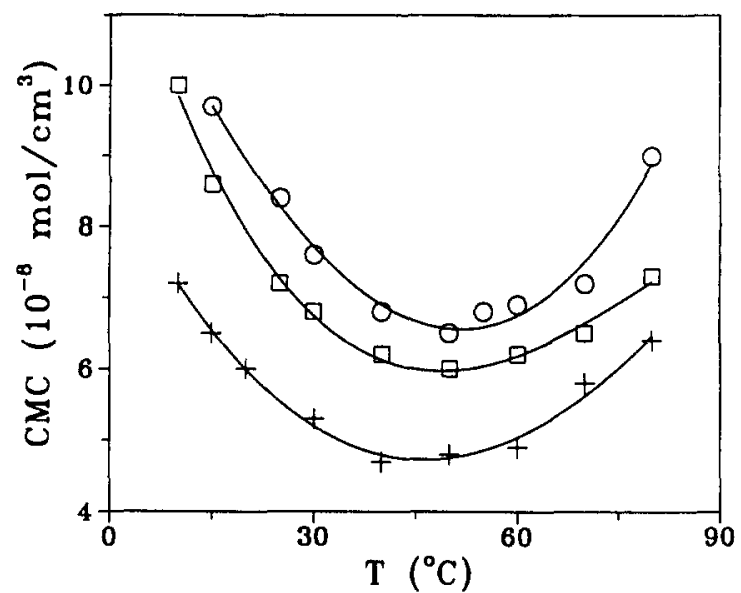

Fig. 2. The variation of $\mathrm{CMC}$ as a function of temperature for the surfactants $C_{12} E_{4}(+), C_{12} E_{6}(\square)$, and $C_{12} E_{8}(O)$.

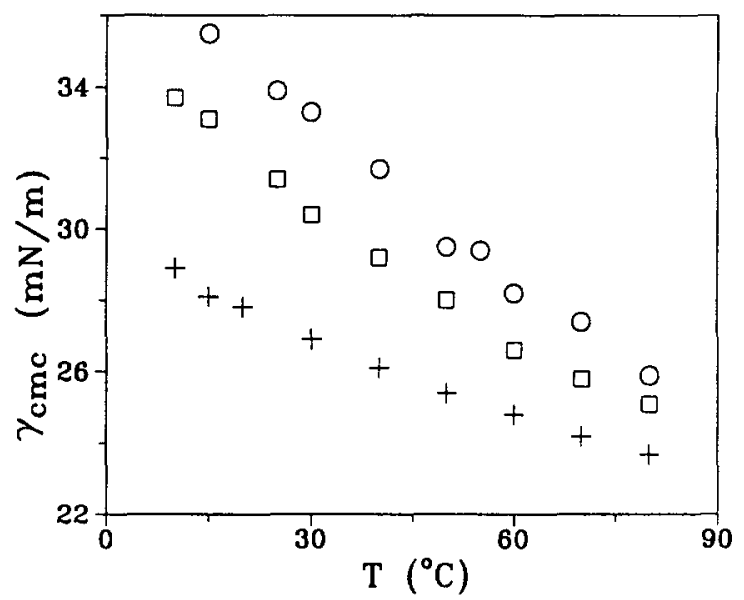

Fig. 3. The variation of surface tension at $\mathrm{CMC} \gamma_{\mathrm{CMC}}$ as a function of temperature for the surfactants $\mathrm{C}_{12} \mathrm{E}_{4}(+), \mathrm{C}_{12} \mathrm{E}_{6}(\square)$, and $\mathrm{C}_{12} \mathrm{E}_{8}(\mathrm{O})$.

water and surfactant molecules. While the temperature increases, some of the existing hydrogen bonds would rupture due to thermal fluctuation, which makes the surfactant molecules more hydrophobic, and thus the surface tension decreases With a longer oxyethylene chain length surfactant, e.g. $C_{12} E_{8}$, the oxyethylene chain is greatly hydrated. Therefore, the dehydration effect would be somewhat more pronounced for longer oxyethylene chain length surfactants since the surfactant molecule binds considerably more water molecules. In addition, the surface pressure $\pi_{\mathrm{CMC}}$, which is 
defined by $\pi_{\mathrm{CMC}}=\gamma_{0}-\gamma_{\mathrm{CMC}}$, is also listed in Tables $\mathbf{1}-3$. The symbol $\gamma_{0}$ is the surface tension of water. Rosen et al. [7] found a decrease of $\pi_{\mathrm{CMC}}$ with rising temperature for $\mathrm{C}_{12} \mathrm{E}_{j} \mathrm{~s}$ with small numbers of oxyethylene groups, whereas with more than five oxyethylene groups $\pi_{\mathrm{CMC}}$ remains almost constant for the temperature ranging from $10^{\circ} \mathrm{C}$ to $40^{\circ} \mathrm{C}$. Fig. 4 shows the variation of surface pressure $\pi_{\mathrm{CMC}}$ over a wide temperature range $\left(10-80^{\circ} \mathrm{C}\right)$ for three surfactants $\mathrm{C}_{12} \mathrm{E}_{4}, \mathrm{C}_{12} \mathrm{E}_{6}$. and $\mathrm{C}_{12} \mathrm{E}_{8}$. For the $\mathrm{C}_{12} \mathrm{E}_{4}$ system, the surface pressure $\pi_{\mathrm{CMC}}$ decreases monotonically with temperature. For the $\mathrm{C}_{12} \mathrm{E}_{6}$ system, the surface pressure $\pi_{\mathrm{CMC}}$ remains constant at low temperatures, and then decreases with further increasing temperature. However, for the $C_{12} E_{8}$ system, the surface pressure $\pi_{\mathrm{CMC}}$ remains almost constant over the whole temperature range, except at high temperature region, a slight decrease of surface pressure $\pi_{C M C}$ is observed. Our results are consistent with those of Rosen et al. [7] in the regime of temperatures below $40^{\circ} \mathrm{C}$. In contrast, a maximum surface pressure $\pi_{C M C}$ was observed for the $\mathrm{OPE}_{j} \mathrm{~s}$ with three to six oxyethylene groups [5].

The temperature dependence of the CMC can be applied to calculate the enthalpy and entropy of micelle formation. According to phase separation model [12] and mass action model [13], the standard Gibbs free energy of micelle formation

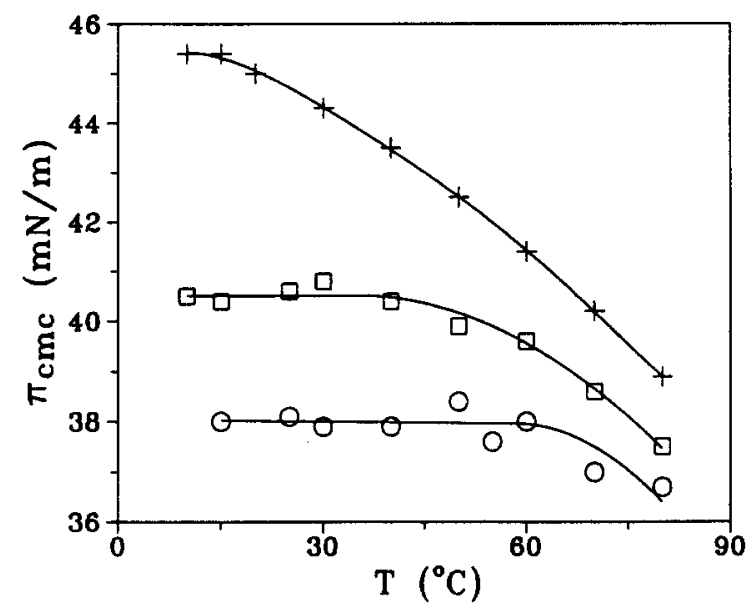

Fig. 4. The variation of surface pressure $\pi_{\mathrm{CMC}}$ as a function of temperature for the surfactants $C_{12} E_{4}(+), C_{12} E_{6}(\square)$, and $\mathrm{C}_{12} \mathrm{E}_{8}(\mathrm{O})$. per mole of monomer is given by

$\Delta G_{\mathrm{m}}^{\mathrm{o}}=R T \ln x_{\mathrm{CMC}}$

where $x_{\text {CMC }}$ stands for the mole fraction of surfactant in aqueous solution at the CMC.

The enthalpy of micelle formation can be obtained by applying the Gibbs-Helmholtz equation to Eq. (1)

$\Delta H_{\mathrm{m}}^{\mathrm{o}}=-T^{2} \frac{\partial\left(\Delta G_{\mathrm{m}}^{\mathrm{o}} / T\right)}{\partial T}=-R T^{2} \frac{\partial \ln x_{\mathrm{CMC}}}{\partial T}$

as long as the aggregation number of monomers per micelle is not too low and not strongly temperature-dependent [14]. To evaluate the enthalpy of micelle formation, the CMCs are first correlated by a polynomial equation

$\ln x_{\mathrm{CMC}}(T)=a+b T+c T^{2}+d T^{3}$

where constants $a, b, c$, and $d$ are determined by least-squares regression analyses. The enthalpy of micelle formation is then calculated numerically by substituting the Eq. (3) into Eq. (2).

$\Delta H_{\mathrm{m}}^{\mathrm{o}}=-R T^{2}\left(b+2 c T+3 d T^{2}\right)$

Once the Gibbs free energy and the enthalpy of micelle formation are obtained, obviously the entropy of micelle formation can be determined by

$\Delta S_{\mathrm{m}}^{\mathrm{o}}=\frac{1}{T}\left(\Delta H_{\mathrm{m}}^{\mathrm{o}}-\Delta G_{\mathrm{m}}^{\mathrm{o}}\right)$

Fig. 5 shows the temperature dependence of $\Delta G_{\mathrm{m}}^{\mathrm{o}}, \Delta H_{\mathrm{m}}^{\mathrm{o}}$, and $-T \Delta S_{\mathrm{m}}^{\mathrm{o}}$ for the surfactants $\mathrm{C}_{12} \mathrm{E}_{4}, \mathrm{C}_{12} \mathrm{E}_{6}$, and $\mathrm{C}_{12} \mathrm{E}_{8}$ in an aqueous system. At low temperatures, the micellization process is endothermic $\left(\Delta H_{\mathrm{m}}^{\mathrm{o}}>0\right)$ and becomes exothermic $\left(\Delta H_{\mathrm{m}}^{\mathrm{o}}<0\right)$ at high temperatures. Our result, Fig. 5, shows that the negative value of Gibbs free energy of micelle formation is mainly due to the large positive value of entropy of micelle formation, especially at low temperatures. Therefore, the micellization is an entropy-driven process.

The entropy change of micellization process is always positive over the whole temperature range. The increase in entropy of micelle formation in an aqueous medium can be explained in two aspects: (1) the iceberg formation [15] of the water mole- 

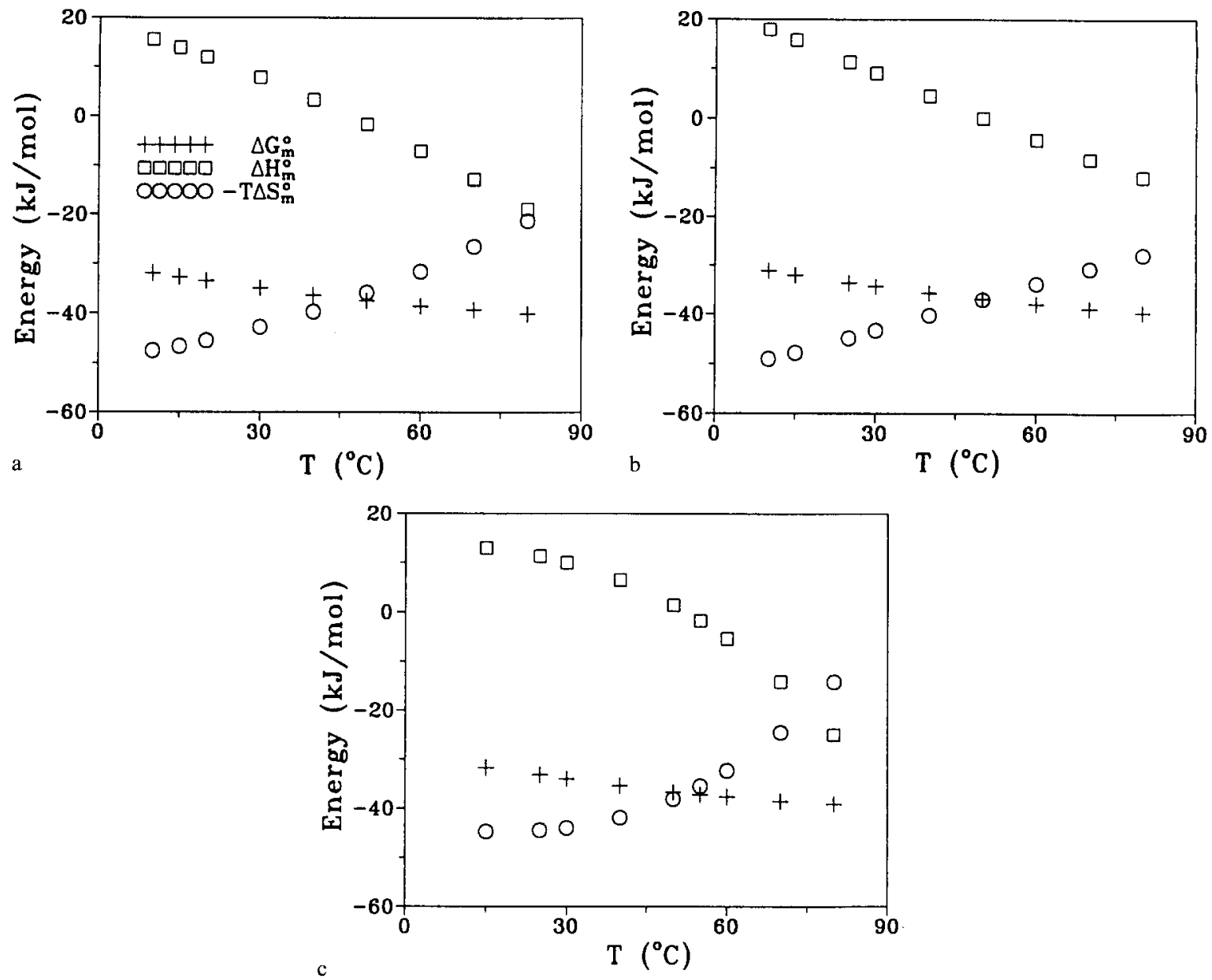

Fig. 5. The variations of $\Delta G_{\mathrm{m}}^{\mathrm{o}}(+), \Delta H_{\mathrm{m}}^{\mathrm{o}}(\square)$, and $-T \Delta S_{\mathrm{m}}^{\mathrm{o}}(\mathrm{O})$ as a function of temperature for the surfactants (a) $\mathrm{C}_{12} \mathrm{E}_{4},(\mathrm{~b})$ $\mathrm{C}_{12} \mathrm{E}_{6}$, and (c) $\mathrm{C}_{12} \mathrm{E}_{8}$ in an aqueous system.

cules surrounding the solute (surfactant) molecules would increase the system "order", then the micellization process by removing the surfactant molecules from the aqueous medium to the micelle would certainly increase the entropy of the system simply due to the rupture of iceberg; (2) the degree of rotational freedom of the hydrophobic chain of surfactant molecules in the non-polar interior of the micelle is much larger than that in the aqueous medium [16]; in other words, the configurational entropy of hydrophobic chain of surfactant molecules is increased when the surfactant molecules are removed from the aqueous medium to the micelle.

It is found that $\Delta G_{\mathrm{m}}^{\mathrm{o}}$ decreases monotonically as the temperature increases over the whole temperature range from $10^{\circ} \mathrm{C}$ to $80^{\circ} \mathrm{C}$. Both $\Delta H_{\mathrm{m}}^{\circ}$ and $\Delta S_{\mathrm{m}}^{\mathrm{o}}$ appear to decrease monotonically with an increase in temperature. According to Eq. (5), these two properties $\left(\Delta H_{\mathrm{m}}^{\mathrm{o}}\right.$ and $\left.\Delta S_{\mathrm{m}}^{\circ}\right)$ have opposite effects on $\Delta G_{\mathrm{m}}^{\mathrm{o}}$. Therefore, $\Delta G_{\mathrm{m}}^{\mathrm{o}}$ depends on the relative amount of the changes in $\Delta H_{\mathrm{m}}^{\mathrm{o}}$ and $\Delta S_{\mathrm{m}}^{\mathrm{o}}$. Fig. 6 shows the enthalpy-entropy compensation plot, i.e. $\Delta H_{\mathrm{m}}^{\mathrm{o}}$ vs. $\Delta S_{\mathrm{m}}^{\mathrm{o}}$ plot. It is interesting to observe the good linearity in the compensation plot, that can be interpreted by [15]

$\Delta H_{\mathrm{m}}^{\mathrm{o}}=\Delta H_{\mathrm{m}}^{*}+T_{\mathrm{c}} \Delta S_{\mathrm{m}}^{\mathrm{o}}$

where $T_{\mathrm{c}}$ is the compensation temperature and $\Delta H_{\mathrm{m}}^{*}$ is the intercept of the compensation line. In 


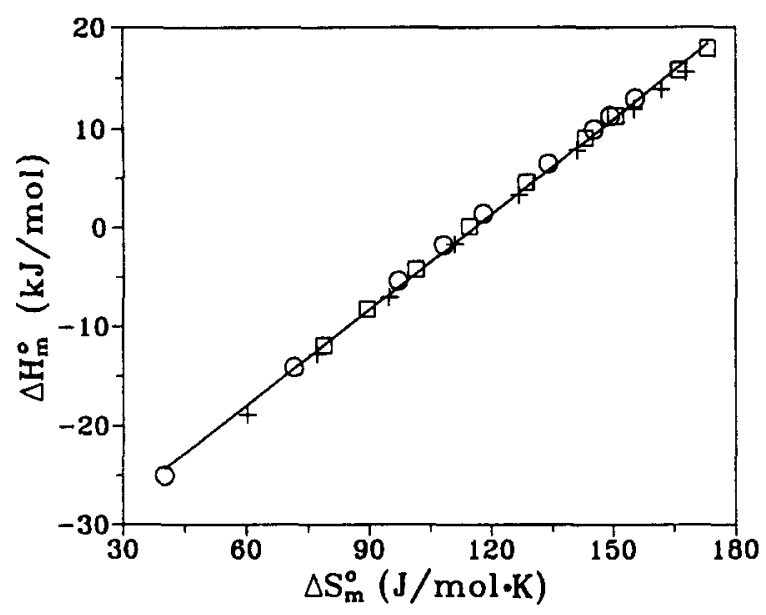

Fig. 6. Enthalpy of micelle formation vs. entropy of micelle formation: $\mathrm{C}_{12} \mathrm{E}_{4}(+), \mathrm{C}_{12} \mathrm{E}_{6}(\square)$, and $\mathrm{C}_{12} \mathrm{E}_{8}(\mathrm{O})$.

fact, the compensation curve of all three surfactants is slightly convex in Fig. 6. In other words, the compensation temperature is temperaturedependent and slightly larger at higher temperatures, in accord with the previous findings $[15,17]$. It is well believed that the compensation temperature is a characteristic of hydrophobic interactions between solvent and solute molecules and the intercept $\Delta H_{\mathrm{m}}^{*}$ is the measure of solute-solute interaction [18]. The compensation temperature is found to be $321 \mathrm{~K}$ by linear regression over the whole temperature range and over all three surfactant systems together.

Over the temperature range $0-30^{\circ} \mathrm{C}$ the compensation temperature of $\mathrm{C}_{12} \mathrm{E}_{6}$ in aqueous solutions was previously found [19] to be $284 \mathrm{~K}$, smaller than our result. It is interesting to note that the compensation temperature of this study is dramatically larger than that of Jolicoeur and Philip [20], who found the compensation temperatures $222 \mathrm{~K}$ and $242 \mathrm{~K}$ for $48 \mathrm{non}$-ionic surfactants in aqueous solutions at $25^{\circ} \mathrm{C}$ and $50^{\circ} \mathrm{C}$ respectively. In contrast, our finding is quite consistent with their compensation temperatures $\sim 315 \mathrm{~K}$ for ionic surfactants in aqueous solutions [20], although $\mathrm{C}_{12} \mathrm{E}_{j} \mathrm{~s}$ are non-ionic surfactants.

\section{Acknowledgment}

This work was supported by the National Science Council of Taiwan, Republic of China.

\section{References}

[1] M.J. Rosen, Surfactants and Interfacial Phenomena, 2nd edition, Wiley, New York, 1989.

[2] B.D. Flockhart, J. Colloid Sci. 16 (1971) 484.

[3] J.A. Stead, H. Taylor, J. Colloid Interface Sci. 30 (1969) 482.

[4] C. La Mesa, J. Phys. Chem. 94 (1990) 23.

[5] E.H. Crook, G.F. Trebbi, D.B. Fordyce, J. Phys. Chem. 68 (1964) 3592.

[6] K. Meguro, M. Ueno, K. Esumi, in: M.J. Schick (Ed.), Nonionic Surfactants, Dekker, New York, 1987, p. 109 and references cited therein.

[7] M.J. Rosen, A.W. Cohen, M. Dahanayake, X. Hua, J. Phys. Chem. 86 (1982) 541.

[8] P. Becher, in: M.J. Schick (Ed.), Nonionic Surfactants, Dekker, New York, 1966.

[9] (a) J.C. Lang, in: V. Degiorgio, M. Corti, Physics of Amphiphiles: Micelles, Vesicles and Microemulsion, North Holland, Amsterdam, 1985. (b) V. Degiorgio, in: V. Degiorgio, M. Corti, Physics of Amphiphiles: Micelles, Vesicles and Microemulsion, North Holland, Amsterdam, 1985.

[10] S.-Y. Lin, R.-Y. Lin, L.-W. Lin, S.-I. Chen, Langmuir 12 (1996) 6530.

[11] R.-Y. Tsay, S.-Y. Lin, L.-W. Lin, S.-I. Chen, Langmuir 132 (1997) 3191.

[12] E. Matijevic, B.A. Pethica, Trans. Faraday Soc. 54 (1958) 587

[13] J.N. Philips, Trans. Faraday Soc. 51 (1955) 561.

[14] J.M. Corkill, J.F. Goodman, S.P. Harrold, Trans. Faraday Soc. 60 (1964) 202.

[15] H.S. Frank, M.W. Evans, J. Chem. Phys. 13 (1954) 507.

[16] G. Stainsby, A.E. Alexander, Trans. Faraday Soc. 46 (1950) 587.

[17] D.J. Lee, Colloid Polym. Sci. 273 (1995) 539.

[18] R. Lumry, S. Rajender, Bipolymers 9 (1970) 1125.

[19] A. Goto, M. Takemoto, F. Endo, Bull. Chem. Soc. Jpn. 58 (1985) 247.

[20] C. Jolicoeur, P.R. Philip, Can. J. Chem. 52 (1974) 1834. 